\title{
Events
}

\section{of Interest}

\section{December 2007}

American College of Chest Physicians (ACCP) Ultrasonography: Fundamentals in Critical Care Meeting: December 7-10, 2007, Hyatt Resort and Spa at Gainey Ranch, Scottsdale, Arizona. For more information contact: ACCP Customer Relations (tel: 800-3432227; E-mail: accp@chestnet.org).

\section{January 2008}

NewEra Cardiac Care 2008:

Innovation and Technology

Course: January 10-13, 2008, Four Seasons Aviara Resort, Carlsbad, California. CME credit pending. Address for information: Aligned Management Associates, Inc., 793-A Foothill Blvd, PMB \#119, San Luis Obispo, CA 93405 (telephone: +1805-534-0300; fax: +1-805-534-9030; Email: info2008@amainc.com; Website: http://www.amainc.com/newerahome.html).

\section{February 2008}

CREF 2008: The San Diego Cardiothoracic Surgery Symposium: Science and Techniques of Perfusion Conference: February 14-17, 2008, San Diego Marriott Hotel \& Marina, San Diego, California. CME credit pending. Abstract deadline: December 30, 2007. Address for information: Aligned Management Associates, Inc., 793-A Foothill Blvd,

Announcements of major meetings and other significant activities must be received at least 8 weeks before the desired month of publication. Information will be limited to title of meeting, date, place, and an address to obtain further information. Send announcements to Pamela W. Fried, Editorial Office, The Journal of Thoracic and Cardiovascular Surgery, Drexel University College of Medicine, 245 N 15th St, Mail Stop 496, Philadelphia, PA 19102-1192 (E-mail: jtcvs@DrexelMed.edu). (telephone: +1-805-534-0300; fax: +1 805-534-9030; E-mail: info2008@amainc. com; Website: http://www.amainc.com/ cref_cardiothoracic.html).

\section{March 2008}

The Society for Cardiothoracic Surgery in Great Britain and Ireland: 2008 Annual Meeting and Forum for Cardiothoracic Surgical Practice Meeting: March 9-12, 2008, Edinburgh International Conference Centre, Edinburgh,
UK. For further information, contact: sctsadmin@scts.org

Interventional Cardiology 2008: 23rd Annual International Symposium Symposium: March 9-14, 2008, Silvertree Hotel, Snowmass Village, Colorado. For more information contact: Promedica International CME, a California Corporation, 2333 State Street, Suite 203, Carlsbad, CA 92008 (telephone: 760-720-2263; fax: 760720-6263; E-mail: education@promedicacme. com; Web site: www.promedicacme.com).

General Thoracic Surgical Club 22nd Annual Meeting

Meeting: March 13-16, 2008, Rancho Bernardo Inn, San Diego, California. For information, please e-mail: blemmerman@ mayo.edu

\section{April 2008}

The Houston Aortic Symposium: Frontiers in Cardiovascular Diseases Symposium: April 4-6, 2008, Houston, Texas. For more information contact: Promedica International CME, a California Corporation, 2333 State Street, Suite 203, Carlsbad, CA 92008 (telephone: 760-720-2263; fax: 760-720-6263; E-mail: education@promedicacme.com; Web site: www.promedicacme.com).

\section{May 2008}

Aortic Symposium 2008 Call for Abstracts

Symposium: May 8-9, 2008, Sheraton New York Hotel \& Towers, New York, NY. Abstract Deadline: December 14, 2007. For more information contact: American Association for Thoracic Surgery, 900 Cummings Center, Suite 221-U, Boston, MA 01915 (telephone: 978-927-8330; fax: 978-524-8890; E-mail: aats@ prri.com; Website: www.atts.org). 
4th International Conference on Pediatric Mechanical Circulatory Support Systems, Pediatric Heart Transplantation, and Pediatric Cardiopulmonary Perfusion

Conference: May 22-24, 2008, Portland Hilton, Portland, Oregon. CME credit available. Abstract submission dead- line: January 31, 2008. Address for information: Penn State College of Medicine CME Department, PO Box 851, Hershey, PA, USA 17033 (telephone: +1 717531 6483; fax: +1 717531 5604; E-mail: continuinged@hmc.psu.edu; Web site: http://www.hmc.psu.edu/ce/ pediatrics).

\section{October 2008}

\section{CHEST 2008}

Annual Meeting: October 25-30, 2008, Philadelphia, Pennsylvania. For more information contact: ACCP Customer Relations (telephone: 847-498-8387; E-mail: accp@chestnet.org). 